\title{
La qualité du sommeil chez les internes : étude transversale chez les internes à l'hôpital Hôtel-Dieu de France (Liban)*
}

\section{Sleep quality of medical students: a cross-sectional study at Hôtel-Dieu de France hospital (Lebanon)}

\author{
Elise SLIM, Mirabelle MATTAR, Grace ABI RIZK \\ Centre hospitalier universitaire Hôtel-Dieu de France, Beyrouth, Liban
}

Manuscrit soumis à la rédaction le 25 juillet 2012 ; commentaires éditoriaux formulés aux auteurs le 27 janvier 2013 et le 6 mars 2014 ; accepté pour publication le 7 mars 2014

\begin{abstract}
Mots-clés Sommeil ; internes ; qualité

Résumé-Objectif : Porter un regard critique sur la qualité du sommeil chez les internes affectés à l'hôpital Hôtel-Dieu de France à Beyrouth (Liban), afin de recenser les différents facteurs pouvant engendrer une perturbation de leur sommeil. Méthodes : C'est une étude transversale qui concerne tous les internes (étudiants de sixième et septième années de médecine). A la fin du mois de décembre 2010, un questionnaire composé de deux parties a été distribué aux internes suite aux cours magistraux. La première partie du questionnaire inclut des questions sur les caractéristiques démographiques, les horaires du travail et l'hygiène du sommeil. La deuxième partie du questionnaire correspond à la version française validée du Pittsburg Sleep Quality Index. Les données ont été analysées par le programme SPSS (Version16.0). Résultats : Parmi les 140 internes sollicités, 101 ont répondu au questionnaire. La prévalence de la mauvaise qualité du sommeil retrouvée chez les internes à l'hôpital Hôtel-Dieu de France est plus élevée que celle retrouvée chez les étudiants en médecine et les résidents en Iran (15\%) et comparable à celle retrouvée chez les étudiants en médecine en Lituanie (40\%) et à Hong-Kong (57,5\%). En analyse bi variée, une relation significative a été trouvée entre la mauvaise qualité du sommeil et le manque de repos après la garde $(p=0,034)$, la consommation élevée de caféine $(p=0,038)$ ainsi que l'utilisation de l'ordinateur le soir $(p=0,007)$. En analyse multi variée, une consommation élevée de caféine et l'utilisation de l'ordinateur restent les deux déterminants significatifs de la mauvaise qualité du sommeil. Conclusion : La prévalence de la mauvaise qualité du sommeil chez les internes à l'hôpital Hôtel-Dieu de France est élevée. La mauvaise qualité du sommeil est corrélée à la consommation élevée de caféine et l'usage de l'ordinateur le soir.
\end{abstract}

\footnotetext{
* Du matériel éditorial complémentaire est disponible en ligne sur la version électronique du présent article.
} 


$\begin{array}{ll}\text { Keywords } & \text { Abstract - Background: Sleep disturbance is growing among interns. Bad sleep hygiene } \\ \text { Sleep; } & \text { and irregular work schedules affect both the quality and length of sleep periods. Sleep disor- } \\ \text { quality; } & \text { ders can affect interns' quality of education, work and life. The objective of the study is to } \\ \text { interns } & \text { determine the prevalence of bad sleep quality among interns. Methods: The cross-sectional } \\ & \text { study involves all interns in Hôtel-Dieu de France hospital. At the end of December } 2010 \text { all } \\ \text { the interns were assessed through a self-administered questionnaire. The first part of the } \\ \text { questionnaire included questions on demographic characteristics, work schedules and sleep } \\ \text { hygiene. The second part was the validated French version of the Pittsburg Sleep Quality } \\ \text { Index. Data was analyzed with the SPSS (version 16.0) program. Chi-2 and Fischer exact } \\ \text { tests were used in the bivariate analysis and logistic multiple regression test for the multiva- } \\ \text { riate analysis. Results: Among the 140 interns involved, 101 filled out the questionnaire. The } \\ \text { prevalence of bad sleep quality found amongst interns at Hôtel-Dieu de France hospital was } \\ \text { much higher than the one found amongst Iranian medical students and residents }(15 \%) \text { and } \\ \text { comparable to that of medical students in Lithuania (40\%) and Hong-Kong }(57.5 \%) \text {. Biva- } \\ \text { riate analysis showed a significant statistical correlation between bad sleep quality and high } \\ \text { levels of caffeine consumption }(p=0.038) \text {, nightly computer use ( } p=0.007) \text { and work shifts } \\ \text { of more than } 30 \text { consecutive hours }(p=0.034) \text {. Multivariate analysis showed that bad sleep } \\ \text { quality was associated with high levels of caffeine consumption and nightly computer use by } \\ \text { interns. Conclusion: Bad sleep quality is highly prevalent amongst interns in Hôtel-Dieu de } \\ \text { France hospital and associated with high levels of caffeine consumption and nightly compu- } \\ \text { ter use. }\end{array}$

\section{Introduction}

La littérature scientifique internationale en santé s'intéresse de plus en plus au sommeil, la somnolence diurne excessive étant un problème de santé publique $^{[1]}$. Le sommeil est essentiel pour la restauration des processus physiologiques qui permettent le bon fonctionnement du corps et de l'esprit ${ }^{[2]}$. C'est le temps où les activités cérébrales traitent les acquisitions de la journée écoulée, tout en facilitant la consolidation des informations dans la mémoire à long terme ${ }^{[3]}$.

Le cycle sommeil/éveil est réglé par deux facteurs majeurs : les facteurs homéostatiques et les facteurs circadiens (rythme circadien) ${ }^{[4,5]}$. Le rythme circadien est l'horloge biologique de l'individu. Il dépend des facteurs suivants : le rythme de sécrétion de la mélatonine hypophysaire (hormone du sommeil), la photo réception circadienne (exposition à la lumière), le rythme des tendances à l'éveil et au sommeil, et le rythme de la température corporelle (la température corporelle étant plus élevée en fin de journée) ${ }^{[5,6]}$. Quant aux facteurs homéostatiques, ils dépendent de la durée du cycle éveil/sommeil de la journée précédente. Les facteurs homéostatiques contrôlent la dette de sommeil ${ }^{[5]}$.

Les conséquences d'un mauvais sommeil, telles qu'elles sont ressenties par l'individu dépendent non seulement de la quantité du sommeil mais aussi de sa qualité et de son horaire ${ }^{[7]}$. L'altération quantitative et qualitative du sommeil retentit sur les performances diurnes ${ }^{[6,8-11]}$. Les risques du mauvais sommeil sont nombreux. Ils incluent un risque plus élevé d'accidents de la voie publique ${ }^{[10]}$, un risque plus élevé d'obésité ${ }^{[11-13]}$, de diabète ${ }^{[14-17]}$, de maladies cardiovasculaires $^{[14,18]}$, de baisse de l'immunité, d'augmentation de la mortalité et de la mauvaise qualité de vie ${ }^{[11,14]}$, ainsi que, sur un autre plan, le risque d'erreur au travail ${ }^{[19]}$.

L'activité des professionnels de la santé met en jeu directement la sécurité des patients. Chez le personnel médical, la privation aigüe et chronique du sommeil aggrave le risque d'erreurs médicales. Cette privation semble être le résultat des longs horaires de travail et des interruptions du rythme circadien par le travail nocturne ${ }^{[7]}$. Des études conduites aux Etats- Unis 
montrent que les erreurs médicales causent plus d'un million d'accidents et peuvent causer la mort de 98000 patients chaque année ${ }^{[9,19]}$. Les internes occupent deux fonctions, celle de stagiaire, dévolue à l'apprentissage et celle de praticien hospitalier, en charge d'activités de soins aux patients à l'hôpital. Dès lors, leur repos est impératif afin de maintenir à la fois leur bien être, la sécurité du patient ainsi que les qualités d'un environnement adéquat à leur éducation.

Au Liban, la qualité du sommeil chez le personnel médical n'a jusqu'à présent pas été étudiée. Le but de cette étude est de documenter la qualité du sommeil chez les internes à l'hôpital universitaire Hôtel-Dieu de France et de porter à cet égard un regard critique, afin de recenser les différents facteurs pouvant engendrer une perturbation de leur sommeil.

De façon corollaire, cette étude cherche à explorer la possibilité d'une alternative au mode de vie que les étudiants mènent, qui pourrait favoriser une meilleure qualité de sommeil, et ultérieurement de meilleures performances. Plus spécifiquement, certains déterminants des habitudes de travail (nombre d'heures du travail par jour, nombre de gardes par mois, prise de repos après la garde et occupation concomitante d'un autre emploi que celui d'interne), de l'hygiène du sommeil (consommation de caféine, consommation d'alcool, présence d'une maladie chronique, temps passé au lit pour des activités non liées au sommeil, usage nocturne de l'ordinateur, pratique nocturne d'exercice physique et prise de siestes diurnes) seront examinés, à la recherche de corrélations positive ou négative entre chaque déterminant et la qualité de sommeil, l'intention étant d'identifier des pistes pour des mesures de prévention ou de réorganisation du temps de travail.

\section{Méthodes}

La population étudiée est constituée des internes de sixième et de septièmes années, affectés à l'hôpital Hôtel- Dieu de France à Beyrouth. Le nombre total des internes est de 140 dont 74 internes en sixième année et 66 internes en septième année.

À la fin du mois de décembre 2010, un questionnaire composé de deux parties a été administré à tous les internes de l'hôpital Hôtel-Dieu de France à Beyrouth, à la suite d'un de leurs cours magistraux. Le questionnaire a été rempli de façon anonyme. Cent et un étudiants ont répondu au questionnaire, soit un taux de réponse de $72 \%$.

\section{Élaboration du questionnaire}

Le questionnaire a été élaboré à partir d'une étude de la littérature scientifique sur le sommeil ; il figure en annexe, disponible en tant que matériel éditorial complémentaire en ligne. Il a notamment pris en compte les critères de l'International classification of Sleep Disorder (ICSD), du manuel diagnostique et statistique des troubles mentaux (Diagnostic and Statistical Manual of Mental Disorders -DSM-IV) et du Pittsburg Sleep Quality Index (PSQI). Le questionnaire comporte deux parties (voir annexe).

La première partie (partie $\mathrm{A}, 21$ questions) recueille les données anamnestiques (cinq questions), les habitudes de travail (six questions) et des informations sur l'hygiène du sommeil (10 questions). Cette partie a été élaborée de novo en se basant sur la revue de la littérature des facteurs perturbateurs du sommeil. Les questions sont des questions à réponse simple. Les questions sur les données anamnestiques concernaient les caractéristiques démographiques, l'année d'internat, la nature du stage effectué pendant le mois écoulé. Les questions sur les habitudes du travail concernaient le nombre d'heures de travail par jour, le nombre de gardes par mois, la prise de repos après la garde et l'occupation concomitante d'un autre emploi que celui d'interne. Les questions sur l'hygiène du sommeil concernaient la consommation de caféine, la consommation d'alcool, la présence d'une maladie chronique, le temps passé au lit pour des activités non liées au sommeil, l'usage nocturne de l'ordinateur, la pratique nocturne d'exercice physique et la prise de siestes diurnes.

La consommation de caféine a été respectivement définie comme étant nulle lorsque l'interne ne consomme aucune tasse de café ni de boisson caféinée, modérée lorsque l'interne consomme moins de trois tasses de café ou moins de six boissons caféinées par jour, et élevée quand l'interne consomme plus de trois tasses de café ou plus de six boissons caféinées par jour. 
La consommation d'alcool le soir a été catégorisée selon trois niveaux, respectivement : aucune consommation, un verre d'alcool et plus d'un verre d'alcool.

La deuxième partie du questionnaire correspond à la version française validée du PSQI ${ }^{[20]}$. Le questionnaire a été administré en français, la version française du PSQI ayant fait l'objet d'une validation psychométrique et sachant que la deuxième langue parlée au Liban est la langue française. De plus, dans l'université à laquelle l'hôpital Hôtel-Dieu de France est affilié, les cours sont offerts exclusivement en français. Enfin, tous les étudiants concernés maitrisent la langue française. Le PSQI consiste en 19 questions d'auto-évaluation et cinq questions posées au conjoint. Seules les questions d'auto-évaluation sont incluses dans le score. Il a été demandé aux participants de limiter leurs réponses aux incidents survenus durant le mois précédent. Cette deuxième partie comporte une seule question fermée sur l'évaluation subjective de la qualité du sommeil (avec cinq choix allant de « très bonne » à « très mauvaise »), 10 questions portant sur la survenue d'un trouble du sommeil (parmi 10 troubles du sommeil), une question sur la somnolence diurne, une question sur la prise de médicament hypnotique (questions fermées avec quatre choix allant d'absence de prise au cours du dernier mois à trois à quatre fois par mois), une question fermée sur leur motivation à pratiquer des activités quotidiennes $(4$ choix allant de «pas de problème » à « très gros problème ») ainsi que quatre questions ouvertes sur l'heure habituelle du coucher, la latence du sommeil, l'heure habituelle du lever et le nombre d'heures du sommeil effectif.

Les questions dans cette partie sont réparties en sept composantes, qui sont respectivement : la qualité subjective du sommeil, la latence du sommeil, la durée du sommeil, l'efficacité habituelle du sommeil, la mauvaise forme durant la journée, l'utilisation d'un médicament qui aide au sommeil et les troubles du sommeil. La somme du score de chacune des sept « composantes » (de 0 à 3 ) constitue le score global du PSQI, qui peut donc varier de 0 à 21 points. Un score de zéro indique l'absence de difficultés, alors qu'un score de 21 indique la présence de difficultés majeures. Selon le PSQI, un total inférieur ou égal à 5 indique une bonne qualité du sommeil. Un total supérieur à 5 permet de poser le diagnostic de mauvaise qualité du sommeil avec une sensibilité de $89,6 \%$ et une spécificité de $86,5 \%$ (kappa $=0,75$, $p<0,001)^{[20]}$.

\section{Méthodes statistiques}

L'étude est de nature transversale avec deux aspects, un aspect descriptif et un aspect analytique. Les données ont été analysées par le programme SPSS data editor -version16.0. Pour la partie analytique, la variable dépendante est la qualité de sommeil, qui est une variable dichotomique (bonne ou mauvaise qualité). Les tests utilisés sont le test du Chi-2 et le test exact de Fisher pour l'analyse bi variée et la régression logistique multiple pour l'analyse multi variée. Le seuil de significativité alpha a été fixé à 0,05.

\section{Résultats}

Les résultats descriptifs

\section{Les données anamnestiques}

Elles sont présentées dans le tableau I. Cinquante trois pour cent des participants sont de sexe féminin et $53,5 \%$ sont des internes en septième année en médecine. Ils sont affectés en stage en majorité dans des services de médecine $(55,45 \%)$ et de chirurgie $(22,77 \%)$.

\section{L'hygiène de travail}

Les données sont présentées dans le tableau I. La majorité des internes travaillent moins de 10 heures par jour $(74,26 \%)$. Tous participants confondus, respectivement $52,48 \%$ et $47,52 \%$ des internes effectuent globalement moins ou plus de quatre gardes par mois. La majorité des internes ne font pas de gardes supplémentaires $(86,14 \%)$. La plupart des internes $(66,33 \%)$ se reposent toujours ou souvent à domicile après la garde. Presque $90 \%$ des internes n'occupent pas d'autre emploi que celui d'interne. 
Tableau I. Les données démographiques et données relatives à l'hygiène du travail. Prévalence et corrélation avec la mauvaise qualité du sommeil.

\begin{tabular}{|c|c|c|c|c|c|}
\hline & & $\begin{array}{c}\text { Prévalence } \\
\%\end{array}$ & $\begin{array}{c}\text { Bonne qualité du } \\
\text { sommeil \% }\end{array}$ & $\begin{array}{c}\text { Mauvaise qualité } \\
\text { du sommeil }\end{array}$ & $P^{*}$ \\
\hline \multirow{2}{*}{ Sexe } & Masculin & $46,5 \%$ & $30(62,5 \%)$ & $18(37,5 \%)$ & \multirow[t]{2}{*}{0,326} \\
\hline & Féminin & $53,5 \%$ & $28(52,83 \%)$ & $25(47,17 \%)$ & \\
\hline \multirow{2}{*}{ Année d'internat } & $6^{\text {ème }}$ année & $46,5 \%$ & $25(53,19 \%)$ & $22(46,81 \%)$ & \multirow[t]{2}{*}{0,422} \\
\hline & $7^{\text {ème }}$ année & $53,5 \%$ & $33(61,11 \%)$ & $21(38,89 \%)$ & \\
\hline \multirow{7}{*}{$\begin{array}{l}\text { Rotation pendant le mois } \\
\text { dernier }\end{array}$} & Médecine & $55,45 \%$ & $31(58,49 \%)$ & $22(41,51 \%)$ & \multirow[t]{7}{*}{$0,662 \#$} \\
\hline & Chirurgie & $22,77 \%$ & $14(60,87 \%)$ & $9(39,13 \%)$ & \\
\hline & Pédiatrie & $8,91 \%$ & $6(66,77 \%)$ & $3(33,33 \%)$ & \\
\hline & Soins/réanimation & $7,92 \%$ & $2(25 \%)$ & $6(75 \%)$ & \\
\hline & Radiologie & $1,98 \%$ & $1(50 \%)$ & $1(50 \%)$ & \\
\hline & anatomopathologie & $2,97 \%$ & $2(66,67 \%)$ & $1(33,33 \%)$ & \\
\hline & Urgences & $2,97 \%$ & $2(66,67 \%)$ & $1(33,33 \%)$ & \\
\hline \multirow{2}{*}{ Horaire du travail/jour } & $<10$ heures/jour & $74,26 \%$ & $43(37,3 \%)$ & $32(42,67 \%)$ & \multirow[t]{2}{*}{0,975} \\
\hline & $>10$ heures/jour & $25,24 \%$ & $15(57,69 \%)$ & $11(42,31 \%)$ & \\
\hline \multirow{2}{*}{ Nombre de gardes/mois } & $\leq 4$ gardes/mois & $52,48 \%$ & $33(62,26 \%)$ & $20(37,74 \%)$ & \multirow[t]{2}{*}{0,301} \\
\hline & $>4$ gardes/mois & $47,52 \%$ & $25(52,08 \%)$ & $23(47,92 \%)$ & \\
\hline \multirow{2}{*}{$\begin{array}{l}\text { Prise de gardes } \\
\text { supplémentaires }\end{array}$} & Non & $86,14 \%$ & $49(56,23 \%)$ & $38(43,68 \%)$ & \multirow[t]{2}{*}{0,576} \\
\hline & Oui & $13,86 \%$ & $9(64,29 \%)$ & $5(35,71 \%)$ & \\
\hline \multirow{2}{*}{$\begin{array}{l}\text { Repos de sécurité a } \\
\text { domicile après la garde }\end{array}$} & Toujours ou souvent & $66,33 \%$ & $44(64,71 \%)$ & $24(35,29 \%)$ & \multirow[t]{2}{*}{$0,034 \$$} \\
\hline & $\begin{array}{l}\text { Quelques fois ou } \\
\text { jamais }\end{array}$ & $32,67 \%$ & $14(42,42 \%)$ & $19(57,58 \%)$ & \\
\hline \multirow{2}{*}{$\begin{array}{l}\text { Travail a temps partiel a } \\
\text { part l'internat }\end{array}$} & Non & $89,11 \%$ & $52(52,78 \%)$ & $38(42,22 \%)$ & \multirow[t]{2}{*}{0,838} \\
\hline & Oui & $10,89 \%$ & $6(54,55 \%)$ & $5(45,45 \%)$ & \\
\hline
\end{tabular}

$* P<0,05$, test de Chi2 sauf \# \$: Valeur statiquement significative \# : Test de Fisher exact

\section{L'hygiène du sommeil}

Les données sont présentées dans le tableau II. La consommation de caféine est élevée dans $18,81 \%$ des cas ; $23,76 \%$ des internes ne consomment pas de caféine et $57,43 \%$ en font une consommation modérée.

La grande majorité de la consommation d'alcool se fait de façon occasionnelle (98\%) : 6,25\% des internes consomment l'alcool comme somnifère ; $52,8 \%$ des internes ne consomment pas l'alcool le soir ; 33,7 \% consomment un verre d'alcool le soir et $13,5 \%$ des internes consomment plus d'un verre d'alcool le soir.
Les maladies chroniques sont présentes chez $5 \%$ des internes. Parmi les internes, 23,76\% pratiquent un exercice physique le soir, $67,33 \%$ passent un temps au lit pour des activités non liées au sommeil et $69,31 \%$ passent un temps sur l'ordinateur le soir. Cinquante huit pour cent des internes prennent des siestes diurnes $(48,28 \%$ moins de deux jours par semaine et $51,72 \%$ plus de deux jours par semaine).

\section{La qualité du sommeil}

Les données sont présentées dans le tableau III. La qualité subjective du sommeil est assez bonne dans 
Tableau II. La prévalence des facteurs affectant la mauvaise hygiène du sommeil, et leur corrélation avec la mauvaise qualité du sommeil.

\begin{tabular}{|c|c|c|c|c|c|}
\hline & & $\begin{array}{c}\text { Prévalence } \\
\%\end{array}$ & $\begin{array}{l}\text { Bonne qualité } \\
\text { du sommeil \% }\end{array}$ & $\begin{array}{c}\text { Mauvaise qualité } \\
\text { du sommeil }\end{array}$ & $P^{*}$ \\
\hline \multirow{3}{*}{ Consommation de caféine } & Nulle & $23,76 \%$ & $16(66,67 \%)$ & $8(33,33 \%)$ & \multirow[t]{3}{*}{$0,038 \$$} \\
\hline & Modérée & $57,43 \%$ & $36(62 \%)$ & $22(37,93 \%)$ & \\
\hline & Elevée & $18,81 \%$ & $6(61,58 \%)$ & $13(68,42 \%)$ & \\
\hline \multirow{3}{*}{$\begin{array}{l}\text { Consommation de l'alcool } \\
\text { le soir }\end{array}$} & Nulle & $52,47 \%$ & $31(58,49 \%)$ & $22(41,51 \%)$ & \multirow[t]{3}{*}{0,472} \\
\hline & 1 verre & $66,67 \%$ & $21(61,76 \%)$ & $13(38,24 \%)$ & \\
\hline & $>1$ verre & $13,86 \%$ & $6(42,86 \%)$ & $8(57,14 \%)$ & \\
\hline \multirow{2}{*}{$\begin{array}{l}\text { Présence de maladie } \\
\text { chronique }\end{array}$} & Non & $95 \%$ & $55(57,29)$ & $41(42,47 \%)$ & \multirow[t]{2}{*}{$1 \#$} \\
\hline & Oui & $5 \%$ & $3(60 \%)$ & $2(40 \%)$ & \\
\hline \multirow{2}{*}{ Exercice physique le soir } & Non & $76,24 \%$ & $45(58,44 \%)$ & $32(41,56 \%)$ & \multirow[t]{2}{*}{0,712} \\
\hline & Oui & $23,76 \%$ & $13(54,17 \%)$ & $11(45,83 \%)$ & \\
\hline \multirow{2}{*}{$\begin{array}{l}\text { Temps au lit le soir pour } \\
\text { des activités non liées au } \\
\text { sommeil }\end{array}$} & Non & $32,67 \%$ & $20(60,61 \%)$ & $13(39,39 \%)$ & \multirow[t]{2}{*}{0,652} \\
\hline & Oui & $67,33 \%$ & $38(55,88 \%)$ & $30(44,12 \%)$ & \\
\hline \multirow{2}{*}{$\begin{array}{l}\text { Temps sur l'ordinateur } \\
\text { avant de dormir }\end{array}$} & Non & $30,69 \%$ & $24(77,42 \%)$ & $7(22,58 \%)$ & \multirow[t]{2}{*}{$0,007 \$$} \\
\hline & Oui & $69,31 \%$ & $34(48,57 \%)$ & $36(51,43 \%)$ & \\
\hline \multirow{2}{*}{ Prise de sieste diurne } & Non & $43 \%$ & $27(62,79 \%)$ & $16(37,21 \%)$ & \multirow[t]{2}{*}{0,348} \\
\hline & Oui & $58 \%$ & $31(53,45 \%)$ & $27(46,55)$ & \\
\hline \multirow{2}{*}{$\begin{array}{l}\text { Nombre de jours/semaine } \\
\text { incluant des siestes diurnes }\end{array}$} & $<2$ jours/semaine & $48,28 \%$ & $16(57,14 \%)$ & $12(42,86 \%)$ & \multirow[t]{2}{*}{0,586} \\
\hline & $>2$ jours/semaine & $51,72 \%$ & $15(50 \%)$ & $15(50 \%)$ & \\
\hline
\end{tabular}

$* P<0,05$, test de Chi2 sauf \# \$: Valeur statiquement significative \#: Test de Fisher exact

Tableau III. La prévalence des composantes de la qualité du sommeil, appréciées selon le Pittsburg Sleep Quality Index (PSQI).

\begin{tabular}{|l|c|c|}
\hline \multirow{2}{*}{ Les sept composantes du PSQI. } & Les déterminants la qualité du sommeil dans le PSQI & $\begin{array}{c}\text { Prévalence } \\
\%\end{array}$ \\
\hline \multirow{2}{*}{ La qualité subjective du sommeil } & Bonne & $71 \%$ \\
\hline \multirow{2}{*}{ La latence du sommeil } & $\leq 3$ Mauvaise & $29 \%$ \\
\hline \multirow{2}{*}{ La durée du sommeil } & $>30$ minutes & $73 \%$ \\
\cline { 2 - 3 } & $>6$ heures & $27 \%$ \\
\hline \multirow{2}{*}{ L'efficacité du sommeil } & $\leq 6$ heures & $83 \%$ \\
\hline \multirow{2}{*}{ La présence de troubles du sommeil } & $\geq 75 \%$ & $17 \%$ \\
\cline { 2 - 3 } & $<7 \%$ & $94 \%$ \\
\cline { 2 - 3 } La prise de médicaments pour le sommeil & Présence de quelques ou plusieurs troubles du sommeil & $13 \%$ \\
\cline { 2 - 3 } & Pas au cours du dernier mois ou <1 fois/semaine & $97 \%$ \\
\hline \multirow{2}{*}{$\begin{array}{l}\text { La sensation de mauvaise forme pendant } \\
\text { la journée }\end{array}$} & Pas au cours du dernier mois ou <1 fois/semaine & $58,5 \%$ \\
\cline { 2 - 3 } & $\geq 1-2$ fois/semaine & $41,5 \%$ \\
\hline
\end{tabular}


Tableau IV. Les facteurs corrélés à la mauvaise qualité du sommeil (analyse multi variée).

\begin{tabular}{|l|c|c|c|c|c|}
\hline & Odds Ratio & Ecart type & $Z$ & $p|z|$ & [Intervalle de confiance -95 \%] \\
\hline Consommation élevée de caféine & 3,65 & 2,466643 & 1,92 & 0,055 & $1-13,7$ \\
\hline Usage de l'ordinateur le soir & 3,24 & 1,625268 & 2,34 & 0,019 & $1,2-8,7$ \\
\hline
\end{tabular}

Modèle a valeur $p=0.0058$ et pseudo $R 2=0.09$.

$55,45 \%$, assez mauvaise dans $24,75 \%$ et très mauvaise dans 3,96\% des cas. La latence du sommeil est comprise entre 0 à 15 minutes dans $39,6 \%$, entre 16 30 minutes dans $33,66 \%$ des cas, entre 31-60 minutes dans $16,83 \%$ des cas et supérieure à 61 minutes dans $9,9 \%$ des cas. La durée totale du sommeil est supérieure à sept heures chez 46,53\% des internes, entre six et sept heures chez $36,63 \%$ des internes, entre cinq et six heures chez $11,88 \%$ des internes et inférieure à cinq heures dans 4,95\% des internes. L'efficacité habituelle du sommeil est en majorité supérieure à $85 \%(81,9 \%)$. Plus de deux tiers des internes $(76,24 \%)$ ont peu de troubles du sommeil, $12,87 \%$ présentent quelques troubles du sommeil et 10,89\% n'en ont pas du tout. La majorité des internes n'utilisent pas de somnifères $(87,13 \%)$. Seule une minorité des internes $(22,77 \%)$ ne fait pas l'expérience d'une «mauvaise forme» durant la journée ou durant la semaine $(35,64 \%) ; 29,7 \%$ en font l'expérience entre une à deux fois par semaine et $11,88 \%$ entre trois à quatre fois par semaine.

\section{Résultats analytiques}

La mauvaise qualité du sommeil (score de PSQI supérieur à 5) a été retrouvée dans $43 \%$ des cas. En analyse bi variée (tableaux I et II), une relation significative a été trouvée entre la mauvaise qualité du sommeil et l'absence de repos de sécurité à domicile après la garde $(p=0,034)$, la consommation élevée de caféine $(p=0,038)$ ainsi que le temps passé sur l'ordinateur avant de se coucher $(p=0,007)$.

En analyse multi variée (tableau IV), une consommation élevée de caféine et l'utilisation nocturne de l'ordinateur restent les deux déterminants significatifs de mauvaise qualité du sommeil, ces deux variables étant responsables de $9 \%$ des cas de mauvaise qualité du sommeil (OR ajustés respectifs $=3,7$ [95\%] de $(1-13,7)$ et $3,2(1,2-8,7), p=0,0058$ et Pseudo $R 2=$ $0,09)$.

La mauvaise qualité du sommeil a été, plus fréquemment, trouvée chez les femmes $(47,17 \%)$ que chez les hommes $(37,5 \%)$, chez les internes de sixième année que chez ceux de septième année (46,81\% vs. 38,89\%), chez les internes affectés dans les services de soins et de réanimation (75\%), chez les internes qui prennent plus de quatre gardes par mois $(47,92 \%)$ que chez ceux qui en prennent moins de quatre $(37,74 \%)$, sans que ces relations soient statistiquement significatives. De même, une tendance à la mauvaise qualité du sommeil est retrouvée chez les internes qui consomment plus d'un verre d'alcool le soir par rapport à ceux qui ne consomment pas d'alcool et ceux qui ne consomment qu' un verre, respectivement $(57,14 \%, 41,51 \%$ et $38,24 \%)$, chez les internes qui passent du temps au lit pour des activités non liées au sommeil $(44,12 \%)$ par rapport à ceux qui ne le font pas $(3,39 \%)$, chez les internes qui prennent des siestes diurnes $(46,55 \%)$ comparés à ceux qui ne le font pas $(37,21$ sans que ces relations soient statistiquement significatives (tableaux I et II).

\section{Discussion}

Aucune étude n'avait jusqu'à présent traité le sujet de la qualité du sommeil de la population générale ni de celle des internes médecins au Liban. Notre étude montre une grande prévalence ( $43 \%$ ) de la mauvaise qualité du sommeil chez les internes affectés à l'hôpital Hôtel-Dieu de France. En analyse multi variée, une 
relation statistiquement significative a été retrouvée entre la mauvaise qualité du sommeil et la consommation élevée de caféine ou l'usage de l'ordinateur le soir.

La mauvaise qualité du sommeil a été définie, comme c'est le cas dans toutes la plupart des études internationales référencées, par un score supérieur à 5 au PSQI, qui est une échelle validée ${ }^{[20]}$.

Les études conduites au Japon, aux États-Unis ou en Europe de l'Ouest montrent des taux d'insomnie allant de 10 à $48 \%$, ces différences traduisant des variations culturelles ou des définitions différentes de l'insomnie $^{[21]}$.

La prévalence de la mauvaise qualité du sommeil chez les internes à l'Hôtel Dieu de France au Liban est beaucoup plus élevée que chez les étudiants en médecine, les résidents et les internes en Iran (où le taux de mauvaise qualité du sommeil est de $15 \%)^{[21]}$. Il faut cependant noter que l'étude iranienne a inclus, outre les internes, des étudiants en médecine moins avancés dans les études, qui pourraient avoir une meilleure qualité du sommeil. La prévalence de la mauvaise qualité du sommeil observée dans la population de cette étude est proche de celle retrouvée chez les étudiants en médecine en Lituanie $(40 \%)^{[22]}$ mais est inferieure à celle retrouvée à Hong-Kong $(57,5 \%)^{[23]}$. Globalement, ces chiffres témoignent de la répartition mondiale du problème.

Notre étude a montré une fréquence de mauvaise qualité du sommeil plus élevée chez les internes de sexe féminin. Dans la littérature, les taux d'insomnie et de fatigue diurne liés à la mauvaise qualité du sommeil sont plus élevés chez les femmes ${ }^{[21,22,24]}$. Le facteur qui peut être le plus incriminé est la présence d'une plus grande susceptibilité aux troubles anxiodépressifs chez les femmes ${ }^{[25,26]}$. Cependant l'absence, dans notre étude, de relation statistiquement significative entre le sexe féminin et la mauvaise qualité du sommeil, peut être expliquée par l'absence, chez les internes de l'hôpital Hôtel-Dieu de France, de différence dans la prévalence de troubles anxio-dépressifs entre les deux sexes. Ceci avait été établi par l'étude, menée au Liban en 2005, de la prévalence des troubles anxio-dépressifs chez tous les étudiants de médecine à l'Université Saint Joseph à laquelle est affilié l'hôpital Hôtel-Dieu de France. Cette étude n' avait, en effet, pas montré de différence de prévalence des troubles anxio-dépressifs selon le sexe ${ }^{[27]}$.
Une mauvaise qualité du sommeil est plus fréquente chez les internes de sixième année que chez les internes de septième année, sans pour autant qu'il $\mathrm{y}$ ait une relation statistiquement significative, ce qui pourrait s'expliquer par les caractéristiques du programme d'études à l'hôpital Hôtel-Dieu de France. En fait, le questionnaire a testé la qualité du sommeil pendant le mois de décembre, au cours duquel les internes de sixième année passent leur premier examen pendant l'internat, ce qui entraine une charge d'études supplémentaire. Toutefois, les internes de septième année passent leur examen de deuxième année d'internat et peuvent alors être exposés à une privation chronique de sommeil et développer une dette du sommeil comparable à celle de leurs collègues de sixième année ${ }^{[28,29]}$, d'où, peut-être l'absence de différence statistiquement significative.

Dans notre étude, la qualité du sommeil n'était pas différente selon la nature du stage d'affectation pendant le mois écoulé. La nature du stage peut affecter le sommeil en raison, soit du nombre de gardes exigées par le service, soit de la charge de travail ou du niveau de stress plus élevé auquel le stage expose (unités de soins intensifs et de réanimation). Une tendance à une plus mauvaise qualité du sommeil a été retrouvée chez les internes affectés en soins intensifs et réanimation que chez ceux en affectés en radiologie ou en médecine. La charge du travail et le stress aux soins intensifs et au service de réanimation sont plus importants que dans d'autres services. De même les gardes en médecine sont plus astreignantes que celles en chirurgie. L'absence de relation statistiquement significative peut être expliquée par la variabilité individuelle dans la perception de l'importance de la charge du travail, des études et du niveau de stress psychique. Le petit nombre des internes en soins intensifs et en réanimation ne permet pas d'établir de comparaison avec leurs pairs dans les différents services.

Il faut souligner que l'hôpital Hôtel-Dieu de France respecte de la charte des internes et les recommandations de l'Accreditation Council for Graduate Medical Education (ACGME) concernant l'horaire du travail. Seuls $26 \%$ des internes travaillent plus de 10 heures par jour, tandis que ce chiffre s'élève à $43 \%$ chez les résidents aux Etats-Unis, qui travaillent plus de 80 heures par semaine ${ }^{[30]}$. La charte des internes ${ }^{[31]}$ recommande un temps de travail de 
10 heures par jour pendant les jours de semaine et quatre heures le samedi, soit un total de 54 heures par semaine. Le respect de la charte à l'hôpital HôtelDieu de France est illustré par l'absence de relation significative entre la mauvaise qualité du sommeil et le nombre d'heures du travail des internes. Il est établi que ce sont les horaires de travail supérieurs à 80 heures par semaine qui sont liés aux troubles du sommeil ${ }^{[30]}$.

La mauvaise qualité du sommeil est plus fréquente chez les internes qui ont plus de quatre gardes par mois, sans que ce soit statistiquement significatif. Il faut souligner que le caractère mesuré de la charge du travail attribuée aux internes (moins de 10 heures par jour chez $74 \%$ ) et le respect du repos de sécurité après la garde (effectif pour $66 \%$ des internes) permet un sommeil récupérateur. La durée du sommeil est supérieure à 6 heures chez $83 \%$, la durée du sommeil inférieure à 6 heures étant celle la plus associée au développement de conséquences du mauvais sommeil $^{[23,35,36]}$.

Onze pour cent des internes occupent un travail à temps partiel en plus de leur emploi d'interne. Ce taux est plus bas que chez les étudiants en médecine et résidents en Iran $(25,7 \%)^{[21]}$, dont la qualité du sommeil était meilleure. A nouveau, on doit souligner que l'étude iranienne inclut des étudiants en médecine qui peuvent avoir plus de temps libre que les internes et résidents. De plus, les internes affectés à l'hôpital Hôtel-Dieu de France proviennent d'une faculté privée ce qui peut être associé à un niveau économique plus élevé.

Une consommation élevée de caféine est positivement liée à une mauvaise qualité du sommeil. Les internes, privés du sommeil, consomment de grandes quantités de caféine, afin de bénéficier de son effet stimulateur $^{[9,28,33,34]}$. Cependant, dans ce cas, la caféine perturbe davantage le sommeil ${ }^{[35]}$. Presque deux tiers des grands consommateurs de caféine ont une mauvaise qualité du sommeil. On note que, selon les critères de l'International classification of sleep disorders (ICSD), la consommation élevée de caféine est considérée comme étant un abus de caféine. Contrairement à l'abus, seule la consommation modérée de substances rentre dans le cadre de la mauvaise hygiène du sommeil ${ }^{[36]}$. Une relation significative n'a pu être relevée entre la consommation modérée de caféine $(200 \mathrm{mg}$ dans la littérature ${ }^{[37]}$ ) et la mauvaise qualité du sommeil.

La moitié des internes consomme de l'alcool le soir mais d'une façon occasionnelle (98\%) et dans un contexte festif $(93,8 \%)$. La mauvaise qualité du sommeil est plus fréquente chez ceux qui consomment plus d'un verre d'alcool le soir mais aussi chez ceux qui ne consomment pas d'alcool le soir, comparativement à ceux qui consomment un seul verre d'alcool le soir. Ces résultats concordent avec les données de la littérature. La consommation d'une grande quantité d'alcool perturbe le sommeil, tandis que la consommation d'un seul verre d'alcool le soir a un effet somnifère ${ }^{[38,39]}$. Toutefois il n'y a pas de relation significative trouvée entre la mauvaise qualité du sommeil et la quantité d'alcool consommée.

Dans notre étude, la pratique d'un exercice physique le soir ne concerne que $23,8 \%$ des internes ; la charge de travail et d'études laisse de fait peu de temps pour l'exercice physique et aucune relation significative n'a été retrouvée entre la mauvaise qualité du sommeil et la pratique d'exercice physique le soir.

Une tendance non significative à la mauvaise qualité du sommeil est trouvée chez ceux qui passent un temps au lit pour des activités non réservées au sommeil. Ceci pourrait être expliqué par le fait que la latence du sommeil chez les internes est inférieure à 30 minutes dans la majorité des cas $(73,26 \%)$. En plus, l'efficacité du sommeil (rapport entre les heures du sommeil effectif et les heures passées au lit) est $>85 \%$ dans $81,19 \%$ des cas. Cette courte latence du sommeil peut être en relation avec la fatigue et la somnolence, causées par d'autres éléments perturbateurs du sommeil, laissant, par la suite, peu de temps au lit pour des activités non liées au sommeil.

Une relation statistiquement significative a été retrouvée entre l'usage de l'ordinateur avant de se coucher et la mauvaise qualité du sommeil. Presque $70 \%$ des internes utilisent l'ordinateur avant de se coucher le soir ; cette fréquence est comparable à celle trouvée dans l'étude espagnole, où $65 \%$ des adolescents utilisent l'ordinateur avant de se coucher et présentent une mauvaise qualité du sommeil ${ }^{[40]}$. Parmi les internes qui utilisent l'ordinateur le soir, $51,4 \%$ présentent une mauvaise qualité du sommeil. 
Dans notre étude, parmi les internes qui prennent des siestes diurnes (58\%), $46 \%$ ont une mauvaise qualité du sommeil, alors que de ceux qui ne prennent pas de sieste diurne, $37 \%$ notent une mauvaise qualité du sommeil. Une tendance non significative à une plus mauvaise qualité du sommeil a été plus souvent retrouvée lors de prises fréquentes de siestes pendant la semaine.

Une partie des résultats de notre étude résulte de l'administration de la deuxième partie du questionnaire, qui correspond à la version française du PSQI qui a fait l'objet d'une validation psychométrique ${ }^{[20]}$. En revanche, bien qu'elle ait été élaborée à partir d'une étude de la littérature scientifique sur le sommeil, ce qui permet de lui attribuer un réel crédit, la première partie du questionnaire n'a pas fait l'objet d'une validation psychométrique formelle, ce qui constitue une limite. D'autres limites sont liées au taux de non-répondants ( $28 \%$ ), dont les caractéristiques spécifiques sont inconnues, et à la petite taille de l'échantillon, qui amoindrit la puissance statistique pour mettre en évidence d'éventuelles corrélations. Par ailleurs, l'utilisation d'une variables catégorique plutôt qu'une variable continue pour la question concernant la consommation de boissons caféinées, a empêché la quantification de la quantité totale de caféine consommée, ce qui a conduit à une perte d'information. Certaines questions additionnelles auraient pu être explorées, telle que celle visant à savoir si les internes cherchent à pallier, avant la garde, la privation du sommeil par des heures de sommeil supplémentaires ${ }^{[32]}$. Enfin, l'effet de l'alcool n'est pas limité au moment du coucher et son effet persiste pendant six heures ${ }^{[38]}$; pour autant, les internes n'ont été interrogés qu'à propos de leur consommation le soir, ce qui peut être à l'origine d'une perte d'informations.

\section{Conclusion}

En dépit de certaines limites, notre étude montre que $43 \%$ des internes affectés à l'hôpital Hôtel-Dieu de France ont une mauvaise qualité du sommeil. Cette prévalence élevée est à mettre en lien avec une mauvaise hygiène du travail (absence de repos à domicile après la garde) et une mauvaise hygiène du sommeil (usage de l'ordinateur le soir), ainsi que par la consommation élevée de caféine. Des études complémentaires devront s'attacher à documenter l'impact de la mauvaise qualité du sommeil sur la qualité du travail.

\section{Contributions}

Elise Slim a participé à la rédaction de l'article, à l'analyse statistique et à la recherche documentaire bibliographique. Grace Abi Rizk a participé à la conception de l'article, à la supervision du travail, à la rédaction et à la révision de l'article. Mirabelle Mattar a participé à la rédaction et à la révision de l'article.

\section{Approbation éthique}

Le projet a été approuvé par le comité d'éthique de la recherche du centre hospitalier universitaire HôtelDieu de France, à Beyrouth (Liban).

\section{Déclaration d'intérêts}

ES déclare n'avoir aucun conflit d'intérêts, MM déclare n'avoir aucun conflit d'intérêts, GAR déclare n'avoir aucun conflit d'intérêts.

\section{Références}

1. Ferrara M, Gennaro LD. How much sleep do we need? Sleep Med Rev 2001;5:155-79.

2. Dworak M, McCarley R, Kim T, Kalinchuk AV, Basheer R. Sleep and Brain Energy Levels: ATP changes during sleep. J Neurosci 2010;30:9007-16.

3. Axmacher N, Haupt S, Fernández G, Elger CE, Fell J. The Role of Sleep in Declarative Memory Consolidation-Direct Evidence by Intracranial EEG. Cereb Cortex 2008;18:500-7.

4. Stevens S. Normal Sleep, Sleep Physiology, and Sleep Deprivation. 2008 [On-line] Disponible sur : http://emedicine.medscape.com/article/1188226overview. 
5. Dijk D, Lockley S. Invited Review: Integration of human sleep-wake regulation and circadian rhythmicity. J Appl Physiol 2002;92:852-62.

6. Collectif. Sommeil. Savoirs et soins infirmiers 2009;60-565-B-10.

7. Weinger M, Ancoli-Israel S. Sleep Deprivation and Clinical Performance. JAMA 2002;287:955-7.

8. Pressman M. Stages and architecture of normal sleep. 2009 UpToDate. [On-line] Disponible sur : http:// www.uptodate.com/contents/stages-and-architecture-of-normal-sleep.

9. Lamberg L. Long Hours, Little Sleep. JAMA 2002;287:303-6.

10. Veasey S, Rosen R, Barzansky B, Rosen I, Owens J. Sleep Loss and Fatigue in Residency Training. JAMA 2002;288:1116-24.

11. Pressman M. Definition and consequences of sleep deprivation. 2009 UpToDate. [On-line] Disponible sur : http://www.uptodate.com/contents/definition-and-consequences-of-sleep-deprivation

12. Gangwisch JE, Malaspina D, Boden-Albala B, Heymsfield S. Inadequate sleep as a risk factor for obesity: analyses of the NHANES I. Sleep 2005;28:1289-96.

13. Hasler G, Buysse DJ, Klaghofer R, Gamma A, Ajdacic $\mathrm{V}$, Eich $\mathrm{D}$ et al. The association between short sleep duration and obesity in young adults: a 13-year prospective study. Sleep. 2004;27:661-6.

14. Buxton O, Pavlova M, Reid E, Wang W, Simonson DC, Adler GK. Sleep Restriction for 1 Week Reduces Insulin Sensitivity in Healthy Men. Diabetes 2010;59:2126-33.

15. Gangwisch JE, Heymsfield SB, Boden-Albala B, Buijs RM, Kreier F, Pickering TG et al. Sleep duration as a risk factor for diabetes incidence in a large U.S. sample. Sleep 2007;30:1667-73.

16. Yaggi H, Araujo A, et McKinlay J. Sleep Duration as a Risk Factor for the Development of Type 2 Diabetes. Diabetes Care 2006;29:657-61.

17. Ayas N, White D, Al-Delaimy W, Manson JE, Stampfer MJ, Speizer FE et al. A Prospective Study of SelfReported Sleep Duration and Incident Diabetes in Women. Diabetes Care 2003;26:380-4.

18. Barger LK, Cade BE, Ayas N, Cronin JW, Rosner B, Speizer FE et al. Extended work shifts and the risk of motor vehicle crashes among interns. N Engl J Med 2005;352:125-34.

19. Jha AK, Prasopa-Plaizier N, Larizgoitia I, Bates DW. Patient safety research: an overview of the global evidence. Qual Saf Health Care 2010;19:42-7.

20. Blais FC, Gendron L, Mimeault V, Morin CM. Evaluation de l'insomnie: validation de trois questionnaires. L'Encéphale 1997;23:447-53.

21. Nojomi M, Bandi F, Kaffashi S. Sleep Pattern in Medical Students and Residents. Arch Iran Med 2009;12:542-9.

22. Preišegolavičiūtè E, Leskauska D, Adomaitienè V. Associations of quality of sleep with lifestyle factors and profile of studies among Lithuanian students. Medicina (Kaunas) 2010;46:482-9.

23. Suen LK, Hon KL, Tam WW. Association between sleep behavior and sleep-related factors among university students in Hong Kong. Chronobiol Int 2008;25:760-75.

24. Arora V, Georgitis E, Woodruff J, Humphrey HJ, Meltzer D. Improving Sleep Hygiene of Medical Interns Can the Sleep, Alertness, and Fatigue Education in Residency Program Help? Arch Intern Med 2007; 167:1738-44.

25. Billiard M, Dauvilliers Y. Insomnie. Elsevier SAS Neurologie 2004;17-025-B-10.

26. Bixler EO, Vgontzas AN, Lin H-M, Have TT, Leiby BE, Vela-Bueno A et al. Respiratory Diseases in Women. European Respiratory Society Monograph 2003;25:204-18.

27. Mehanna Z, et Richa S. Prévalence des troubles anxiodépressifs chez les étudiants en médecine : Étude transversale chez les étudiants en médecine de l'Université Saint-Joseph de Beyrouth. L'Encéphale 2006;32(6P1):976-82.

28. Gaba D, Howard S. Fatigue among Clinicians and the Safety of Patients. N Engl J Med 2002;347:1249-55.

29. Barger L, Cade B, Ayas N, Cronin JW, Rosner B, Speizer FE et al. Extended Work Shifts and the Risk of Motor Vehicle Crashes among Interns. N Engl J Med 2005;352:125-34.

30. Landrigan C, Barger L, Cade B, Ayas NT, Czeisler CA. Interns' Compliance With Accreditation Council for Graduate Medical Education Work-Hour Limits. JAMA 2006;296:1063-70.

31. Comité des stages. Charte des internes. Beyrouth : Université Saint-Joseph, Faculté de médecine, 2008. 
32. Arora V, Georgitis E, Woodruff J, Humphrey H, Meltzer D. Improving Sleep Hygiene of Medical Interns Can the Sleep, Alertness, and Fatigue Education in Residency Program Help? Arch Intern Med 2007; 167:173844.

33. Kiernan M, Civetta J, Bartus C, Walsh S. 24 Hours OnCall and Acute Fatigue No Longer Worsen Resident Mood Under the 80-Hour Work Week Regulations. Current Surgery 2006;63:237-41.

34. Kuo A. Resident overnight call — an idea past its time? West J Med 2001;174:180-1.

35. Sinanian A, Edel Y, Pirlot G, Cupa D. Clinique possible d'une addiction à la caféine à partir de l'observation de 52 sujets et d'une revue de la littérature. Annales médico-psychologiques 2010;168:495-501.

36. American Academy of Sleep Medicine. International classification of sleep disorders, revised: Diagnostic and coding manual. Chicago (IL): American Academy of Sleep Medicine, 2001.
37. Lande R, Reed W. Caffeine-Related Psychiatric Disorders. 2009 [On-line] Disponible sur : http://emedicine.medscape.com/article/290113

38. National Institute on Alcohol Abuse and Alcoholism Alcohol. Alert publication 1998, ${ }^{\circ} 41$ Alcohol and sleep. 1998 [On-line] Disponible sur: http:// pubs.niaaa.nih.gov/publications/aa41.htm

39. Conroy D, Culebras A. Sleep disorders associated with alcohol use and abuse. Medlink neurology 2010 [On-line] Disponbile sur : http://www.medlink.com/medlinkcontent.asp.

40. Mesquita G, Reimao R. Nightly use of computer by adolescents: its effect on quality of sleep. Arq NeuroPsiquiatr 2007;65:428-2.

Correspondance et offprints : Mirabelle Mattar : SUNY Upstate Medical University, 4174 Tommys Trail. 13090 Liverpool (NY), USA. Téléphone : +13152782987 .

Mailto : mirabellemattar@gmail.com 